\title{
Erratum
}

\section{Sarcome de Kaposi buccal post-transplantation rénale : présentation d'un cas et revue de la littérature}

Wafa Hasni ${ }^{1}$, Abdelfatteh Slama ${ }^{2}$, Wissal Sahtout ${ }^{3}$, Moncef Mokni ${ }^{4}$, Habib Kochtali ${ }^{2}$

1 Service de Médecine dentaire, CHU Farhat Hached, Sousse, Tunisie

2 Service de Chirurgie maxillo-faciale, CHU Sahloul, Sousse, Tunisie

3 Service de Néphrologie, Dialyse et Transplantation rénale, CHU Sahoul, Sousse, Tunisie

${ }^{4}$ Laboratoire d'Anatomie pathologique, CHU Farthat Hached, Sousse, Tunisie

(Med Buccale Chir Buccale 2013;19:173-82, D0I: 10.1051/mbcb/2013070)

Il existe une erreur dans l'article original, le nom du premier auteur est Wafa Hasni.

L'article corrigé est disponible en document supplémentaire sur le site de la revue. 